\title{
Diacronie
}

Studi di Storia Contemporanea

$\mathrm{N}^{\circ} 35,3 \mid 2018$

Gli strumenti di Clio

\section{Per un bilancio critico del dibattito sorto intorno a Un'età contro la storia. Saggio sulla rivoluzione del XXI secolo}

\section{Giuseppe Carlo Marino}

\section{(2) OpenEdition \\ Journals}

Edizione digitale

URL: http://journals.openedition.org/diacronie/9139

DOI: 10.4000/diacronie.9139

ISSN: 2038-0925

Editore

Association culturelle Diacronie

Notizia bibliografica digitale

Giuseppe Carlo Marino, « Per un bilancio critico del dibattito sorto intorno a Un'età contro la storia.

Saggio sulla rivoluzione del XXI secolo », Diacronie [Online], № 35, 3 | 2018, documento 13, Messo online

il 29 septembre 2018, consultato il 21 avril 2019. URL : http://journals.openedition.org/diacronie/9139 ; DOI : 10.4000/diacronie.9139 


\title{
Diacronie
}

Studi di Storia Contemporanea

\section{$35,3 / 2018$}

Gli strumenti di Clio: uomini, luoghi e teorie della storia dalla tradizione critica alla comunicazione digitale

\section{Per un bilancio critico del dibattito sorto intorno a Un'età contro la storia. Saggio sulla rivoluzione del XXI secolo}

\author{
Giuseppe Carlo MARINO
}

Per citare questo articolo:

MARINO, Giuseppe Carlo, «Per un bilancio critico del dibattito sorto intorno a Un'età contro la storia. Saggio sulla rivoluzione del XXI secolo», Diacronie. Studi di Storia Contemporanea : Gli strumenti di Clio: uomini, luoghi e teorie della storia dalla tradizione critica alla comunicazione digitale, 35, 3/2018, 29/09/2018,

URL: < http://www.studistorici.com/2018/09/29/marino_numero_35/ >

Diacronie Studi di Storia Contemporanea $\rightarrow$ http://www.diacronie.it

Rivista storica online. Uscita trimestrale.

redazione.diacronie@hotmail.it

Comitato di direzione: Naor Ben-Yehoyada - João Fábio Bertonha - Christopher Denis-Delacour - Maximiliano Fuentes Codera Anders Granås Kjøstvedt - John Paul Newman - Deborah Paci - Niccolò Pianciola - Spyridon Ploumidis - Wilko Graf Von Hardenberg Comitato di redazione: Jacopo Bassi - Luca Bufarale - Gianluca Canè - Fausto Pietrancosta - Alessandro Salvador - Matteo Tomasoni Diritti: gli articoli di Diacronie. Studi di Storia Contemporanea sono pubblicati sotto licenza Creative Commons 3.0. Possono essere

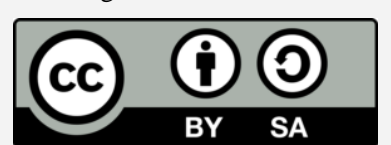
riprodotti e modificati a patto di indicare eventuali modifiche dei contenuti, di riconoscere la paternità dell'opera e di condividerla allo stesso modo. La citazione di estratti è comunque sempre autorizzata, nei limiti previsti dalla legge. 


\title{
13/ Per un bilancio critico del dibattito sorto intorno a Un'età contro la storia. Saggio sulla rivoluzione del XXI secolo
}

\author{
Giuseppe Carlo MARINO
}

Giuseppe Carlo Marino nel suo commento al dibattito sorto intorno al suo saggio su globalizzazione e crisi del "pensare storico" replica agli studiosi che hanno preso parte alla tavola rotonda ripercorrendo i principali nodi teorici proposti e sviluppando gli spunti e i temi emersi nei diversi interventi. Attraverso un approfondimento delle principali questioni trattate nel saggio Marino suggerisce interessanti visioni e offre innovative letture del mondo contemporaneo alla luce degli eventi e delle evoluzioni politiche, economiche e culturali della storia più recente.

\section{Introduzione}

Sei commenti, ovvero sei stimolanti e assai colte letture, sono già un consistente risultato critico del dibattito promosso dalla redazione di questa Rivista aprendo una "tavola rotonda" sui temi del mio recente pamphlet, apparso in provvisoria edizione digitale ${ }^{1}$. Ho da esserne molto grato agli autori, mentre mi auguro che il dibattito, una volta aperto, possa continuare. Intanto, approfitto dell'occasione concessami sia di commentare io stesso il mio scritto, così riletto a distanza di qualche tempo, sia di commentare i commenti. Registro subito con soddisfazione il fatto che i miei commentatori - colleghi anziani e autorevoli, insieme a giovani appartenenti alle nuove leve del lavoro storiografico - siano rappresentativi di diverse generazioni di studiosi. Tra loro, soprattutto i più giovani sono stati davvero molto pazienti con la loro attenzione ad uno scritto il cui dettato, per quanto intenzionalmente destinato a fini di pubblico impegno civile, e certamente estraneo alle correnti forme accademiche (lo direi un saggio corsaro), rimane piuttosto arduo e sembra confinato, così come giudicano gli editori per un autore non molto noto ai media, soltanto ad elitarie letture. Sono ormai lontani gli anni (penso in particolare a quel

\footnotetext{
${ }^{1}$ MARINO, Giuseppe Carlo, Un'età contro la storia. Saggio sulla rivoluzione del XXI secolo, Palermo, Università degli Studi di Palermo, Dipartimento di Scienze Politiche e delle relazioni internazionali (DEMS), 2017, URL: $<$ https://www.unipa.it/dipartimenti/dems/.content/documenti/pubblicazioni/E-Book-Marino-30ottobre.pdf>, [consultato il 3 settembre 2018].
} 
prodigioso 1968 e ai successivi anni Settanta) nei quali persino i testi più ostici potevano essere destinati, e con successo, a letture di massa.

Oggi è il tempo, oltre che di un'eccezionale confusione, anche di una quasi normale disattenzione. A maggior ragione, se ci si muove a sfida della banalità, affrontando tematiche che impongono un forte impegno teoretico. Se si proviene dall'orizzonte di idee e di eventi del Novecento, si può ben avvertire la sgradevole sensazione di dover procedere a tentoni in partibus infidelium o di navigare in un mare ignoto, dopo aver bruciato alle spalle i vascelli della precedente navigazione. Avere acquisito come il sottoscritto (fin dall'anno 2000, con il saggio sull'Eclissi del principe e crisi della storia, edito da Franco Angeli) ${ }^{2}$ la consapevolezza di stare nel vivo di un cambiamento epocale segnato da radicali processi rivoluzionari è una condizione piuttosto rara, poiché di norma delle rivoluzioni (a maggior ragione di quelle "strutturali") non ci si avvede finché sono ancora in fase di svolgimento. E quasi supera i limiti dell'azzardo già il voler delineare per l'interpretazione storiografica - è forse la prima volta che si osa tanto - un unitario quadro periodizzante del contemporaneo che colloca tra gli anni Ottanta del Novecento (con una forte accelerazione nel fatidico anno 1989) e i nostri giorni la cesura con la lunga età, almeno bisecolare, della "rivoluzione industriale" e il passaggio ad un'inedita fase storica (rispetto alla precedente non di mera continuazione, ma appunto di superamento e di integrale "rottura") che ho denominato, "rivoluzione elettronico-informatica": una fase, un nuovo sistema di egemonia in formazione (se si vuole, una nuova grande "rete di significato" per il mondo) che i criteri marxisti di quella che Gramsci chiamava la "scienza della prassi" rendono ben identificabile senza che però essi possano ancora riuscire a suggerire qualcosa di affidabile circa i concreti esiti che ne scaturiranno in futuro. Sembra sensato ipotizzare (un eccentrico storico come Yuval Noah Harari, in Homo deus, ${ }^{3}$ l'ha già fatto!) che il futuro prossimo in fieri sia l'avvio di un nuovo stadio dell'evoluzione umana al di là dell'homo sapiens, verso una nuova e super umanità. Per quanto l'argomento sia tra quelli che si è soliti definire "visionari", è certo opportuno che anche gli storici comincino a prenderlo in seria considerazione, specie se giovani e particolarmente capaci di muoversi in un mondo sempre più dominato dagli algoritmi informatici. A distanza di oltre un secolo Nietzsche torna ad intrufolarsi nel cantiere degli storici.

\section{Commento}

Nel mio pamphlet seguendo il filo dinamico di un lungo passato per poi ancorarmi nell'analisi al quadro attuale del capitalismo globalizzato uscito vincitore dalla "guerra fredda" e resosi capace di instaurare una sorta di "totalitarismo senza confini" - ho comunque inteso inaugurare

\footnotetext{
${ }^{2}$ MARINO, Giuseppe Carlo, Eclissi del principe e crisi della storia. Apogeo e tramonto della democrazia rivoluzionaria nel XXI secolo, Milano, Franco Angeli, 2000.

${ }^{3}$ HARARI, Yuval Noah, Homo deus: breve storia del futuro, Milano, Bompiani, 2017.
} 
un critico avvertimento circa un viaggio già da qualche decennio cominciato in mare aperto, dalle mete imprevedibili, che rende quasi inutilizzabili o almeno desuete le bussole in uso nel passato. Niente di più. E, nell'osservare e nel vivere quanto di sconvolgente sta accadendo (nell'economia, nella società, nella cultura e nella politica), è da assumere come un'indicazione problematica quel filo conduttore della mia interpretazione della rivoluzione in corso che attribuisce al "virus" della tecnologia (ovvero alla sempre più netta subordinazione all'utilità, non solo del lavoro, già reso alienato dal capitalismo, ma anche della scienza) il ruolo di principale forza destruens della vecchia egemonia e insieme di motore o forza construens di quella nuova in formazione.

Nient'affatto problematica, invece, è forse la mia rilevazione (che vedo qui in vario modo condivisa, e comunque non contestata) del principale effetto del primato conseguito dalla tecnologia sulla cultura e sulla stessa scienza: l'eclissi della "ragione dialettica" (ovvero della logica dialettica di cui Hegel, e poi Marx e la scuola marxista, con peculiare rilievo del nostro Gramsci, avevano indicato la consustanzialità con l'humanitas nel suo costitutivo conflitto con la natura che fonda e fa la storia) a tutto vantaggio di una ratio formale-matematica, la logica che presiede agli algoritmi dell'informatica e guida un'incalzante corsa all'innovazione che brucia registrandone l'irrilevanza (se non per un eventuale intrattenimento ludico della memoria riservato a storici-letterati o soltanto a generici curiosi dediti al diletto dell'erudizione!) - tutto il passato dell'homo sapiens che abbiamo fin qui conosciuto e tende ad un superumano collimante con le potenzialità illimitate già intravedibili per l'“intelligenza artificiale". In breve, adesso, riesaminando quanto ho scritto, ritrovo tutta la temerarietà degli argomenti dei quali mi sono avvalso, in un contesto di analisi forse troppo schematico, per lanciare il mio modello interpretativo di "un'età contro la storia". Essendomi esposto a rigorose critiche e a dinieghi affollando le mie pagine di tanti provocatori spunti per il dibattito su molti temi cruciali, non potrei che essere quasi sorpreso nel rilevare che i consensi ricevuti finora dai commenti superano di gran lunga i dissensi. Certo - ed era ben prevedibile - non mancano contrasti e divergenze tra gli stessi commentatori. Il che assumo a riprova di un reale dibattito e ad auspicio per la sua prosecuzione con altri interventi (sono stati promessi e forse verranno).

In un testo qui e là segnato da accenti di amarezza, Francesco Barbagallo condivide con me, da esimio storico della politica e della società, l'allarme per l'eclissi della storicità e ne fa risalire le cause all'egemonia del pensiero unico particolarmente evidenziatosi dopo il fatale 1989 ("l'attuale ideologia diffusa dal dominante capitale finanziario"). Di contro, Giovanni Gozzini (che della questione ha soprattutto presenti i dati di fatto che rendono oggi disagevole il lavoro degli storici di mestiere), mi rimprovera "un eccesso di reductio ad unum" che trova nella mia "identificazione tra globalizzazione e neoliberismo". Egli è certamente il più "specialista" tra i miei commentatori, essendo autore autorevole di specifiche ricerche e riflessioni sulla globalizzazione e, in particolare, di quelle, assai ricche, del suo recente (2018) libro laterziano sul mondo attuale, 
intitolato L'età del disordine $e^{4}$ Ma mi scuserà se ho da ritenere che le sue critiche, per quanto espresse da "sinistra" e persino con richiami a Marx (un tempo si sarebbero dette "socialdemocratiche", oggi forse meglio, soltanto liberal o liberal-riformiste) siano del tutto "interne" proprio a quel pensiero unico della globalizzazione capitalistica dal quale, invece Barbagallo ed io vorremmo fuoruscire. Da altri commenti, specie da quelli dei più giovani, traggo soprattutto importanti stimoli a perfezionare e ad ampliare l'analisi del pamphlet (se mi sarà consentito, in una qualche edizione cartacea). Così dai testi di Carmelo Albanese e di Paola Bernasconi, l'uno mio apprezzato allievo (adesso, qui, gli devo un'esemplare sintesi del mio pamphlet), l'altra un'inattesa rivelazione di partecipe sensibilità critica e di comune moralità di mestiere, esito di una certa fortuna dei miei scritti sulle "generazioni politiche" in Italia"

Ora, mentre è leale che io dichiari la mia soddisfazione soprattutto per i pieni consensi ricevuti, è opportuno che nel rispondere dedichi il maggiore spazio proprio a quei commenti nei quali registro una maggiore lontananza dalla mia riflessione. Le osservazioni critiche del giovane studioso Sebastiano Taccola (di cui ammiro l'acribia e la versatilità teoretica) mi inducono a ritornare su un nodo fondamentale del mio discorso, quello del crollo della "ragione dialettica". Si tratta, a mio avviso, di un processo che ha le sue premesse nel lento abbandono novecentesco del monumentale impianto del pensiero hegeliano con tutte le inevitabili conseguenze anche per l'originario dettato marxista che ne discendeva. Un processo multiforme, frastagliato, e discontinuo, rilevabile, appunto, negli sviluppi del pensiero che si è comunque confrontato con il marxismo nel XX secolo. Se ne sono sviluppati sia un certo anomalo marxismo, sia certi orientamenti "scientisti" a suo superamento, in vario modo dediti a liberarsi dallo "scomodo" Hegel. Su questa strada di intrecci deformanti, richiamerei Althusser e il pensiero dello strutturalismo (da Lévi-Strauss a Foucault e Lacan), nonché certe diffuse influenze della filosofia di Karl Popper ${ }^{6}$. Nella storiografia, il divorzio dalla dialettica hegeliano-marxiana è stata segnata soprattutto dal connubio con lo "strutturalismo" inaugurato e sviluppato dagli storici della grande scuola francese delle Annales. Adesso, qui, nel suo commento, Tacolla, nel muovermi - da un punto di vista che rivendica una certa continuità marxista - delle interessanti critiche, si appella proprio alla fertilità storiografica dello strutturalismo richiamando in proposito Fernand Braudel e la Scuola annalistica ${ }^{7}$. Non riuscirei mai a seguirlo nelle sue predilezioni, se non altro perché proprio lo strutturalismo, e pertanto Braudel e la sua Scuola, mi accertano quanto sia ormai pervasiva nella storiografia quella tendenza alla rimozione del concetto stesso di

\footnotetext{
${ }^{4}$ DETTI, Tommaso, GOZZINI, Giovanni, L'età del disordine: storia del mondo attuale 1968-2017, Roma-Bari, Laterza, 2018.

${ }^{5}$ MARINO, Giuseppe Carlo, Le generazioni italiane dall'Unità alla Repubblica, Milano, Bompiani, 2006.

${ }^{6}$ Si vedano i saggi di ALTHUSSER, Louis, Lenin e la filosofia; Sul rapporto fra Marx e Hegel; Lenin di fronte a Hegel, Milano, Jaca Book, 1972; MANCINA, Claudia (a cura di), Louis Althusser, Freud e Lacan, Roma, Editori riuniti, 1977.

${ }^{7}$ Si veda WALLERSTEIN, Immanuel, «Braudel, le "Annales" e la storiografia contemporanea», in Studi Storici, $21,1 / 1980$, pp. 5-17.
} 
"dialettica", impareggiabilmente definito da Hegel, senza del quale l'humanitas viene integralmente assorbita dalla natura e si smarriscono il senso e il valore specifico dell'operare dell'uomo nel tempo per superare lo stato naturale e sviluppare la civiltà tramite le fatiche e le contraddizioni del lavoro: appunto, quella tendenza "a-dialettica", se non addirittura "antidialettica" che oggi è diventata un risultato; secondo la mia analisi, la radice dell'odierna egemonia che mette in crisi la storia e il "pensare storico" (dato che la natura di per se stessa non ha storia). Naturalmente sono consapevole di quanto sia oggi fuori moda ribadire e tenere ferma una lettura di Marx fondata su Hegel, ma sono anche convinto che non se ne possa prescindere per continuare a pensare storicamente. Se questo non accade (e purtroppo oggi c'è da esserne quasi certi), non si pensa più storicamente, così come il mio pamphlet denunzia. Infatti, nella pur splendida produzione storico-letteraria di Braudel e della scuola annalistica francese, il "pensare storico" tende a convertirsi, con criteri e metodi da "scienza della natura" in una mera analisiricostruzione antropologica del passato: all'acuto Taccola obietterei, pertanto, che è proprio la storiografia "strutturalista" quella che, per usare il linguaggio della sua obiezione, "naturalizza il tempo storico"; ma meriterebbe una più articolata risposta. Me ne astengo per esigenze di spazio e passo oltre.

Certo, a seconda dei punti di vista - se si usassero vecchie, novecentesche categorie di giudizio - il mio pamphlet potrebbe essere considerato sia un testo di radicale innovatività, sia un testo inconsapevolmente "reazionario" (fra poco ne spiegherò il perché). Sono soprattutto grato ad Eugenio Guccione perché con il suo commento mi accerta che ancora ha un senso dirsi di "destra" o di "sinistra" e che io, da antico studioso militante, posso star tranquillo di aver salvato la mia anima di "sinistra". Egli, infatti, scontata la sua delicata gentilezza, e non senza qualche cenno di parziale apprezzamento, mi sembra che manifesti un pressoché integrale disaccordo, pur avendo avuto un ruolo importante - da direttore della rivista "Storia e politica" - per la pubblicazione on-line del saggio (il che me lo conferma in amicizia e in apprezzabile liberalità). Di fronte ai suoi argomenti accetto di proclamarmi in totale disarmo. C'è, infatti, un'impossibilità di comunicare con linguaggio condiviso, tra la dialettica della ragione laica (che mi sembra sia quella propria del pensare storico, anche per chi da "cattolico" voglia continuare a dirsi uno storico) e il dogmatismo religioso assunto a criterio di interpretazione e di giudizio, per quanto alimentato da un rispettabile credo nella "trascendenza", che Guccione - appunto - rivendica a fronte delle concezioni "immanentistiche". Ho da ritenere che in questo modo egli intenda argomentare soprattutto da "filosofo"; del resto, essendo stato io stesso a dargliene occasione nei primi quattro paragrafi del mio saggio con una temeraria incursione, da storico un po' bizzarro, nel territorio delle competenze filosofiche. Ma mi consenta di fargli notare che nell'avere preso le mosse per la mia analisi dal richiamo del mito biblico dell'Eden ben sapevo di indulgere ad una bizzarria letteraria: farne la metafora di un assai improbabile originario "stato di natura" dell'uomo (e 
della "natura" tout court) contrapposto dialetticamente alla reale condizione storica del vivere e del pensare dell'uomo per interpretare e dominare la "natura" appunto nel tempo storico; condizione storica della quale mi sembra che la rivoluzione in corso stia invertendo la rotta sulla linea anti-dialettica della tecnologia, sì da smarrire il senso stesso della storicità per una specie di disumanante "ritorno all'Eden". Ora, Guccione sembra rimproverarmi di non aver attribuito all'Eden altro valore che quello di un mito (quasi che i miti siano soltanto mere fantasie senza valore culturale); mentre per lui non di un mito si tratterebbe, bensì, forse, di una verità certificata dal dettato divino della Bibbia. Ebbene, potrei ritenere invidiabile la sua fede, però scusandomi di non avvertire alcuna propensione a misurarmi con lui sulla faccenda fino al punto di trascendere addirittura nella teologia. Intanto, gli devo particolare considerazione perché il suo commento mi aiuta a capire come e perché uno scritto, che con tutti i suoi limiti è uno scritto marxista sul filo della lezione gramsciana, è risultato tale da indurre se non all'approvazione, almeno all'interessamento, il più onesto pensiero reazionario. In questo, il collega Guccione non è il solo. Infatti, le mie riflessioni in qualche modo inducono ad un'alleanza generazionale quanti abbiano vissuto insieme, poco importa se con idee avverse, un passato di cui costatano la "rottura" e il pressoché definitivo superamento. Sicché è proprio l'avvertimento di una comune condizione di "reduci", destinati comunque a riflettere testimoniando e a testimoniare riflettendo, quel che propizia una concordia discors nel travaglio di confrontare il presente con il passato; e i più arroccati al passato, con la loro drammatica nostalgia per un ancient régime di credenze e valori ormai travolti, sono indotti ad apprezzare tutti gli argomenti che appaiano di critica integrale alla rivoluzione in corso, si tratti pure di argomenti di cui un tempo si sarebbe soltanto diffidato perché propri degli avversari (nel caso in esame, di un avversario marxista come il sottoscritto). Naturalmente così, se può nascere un atteggiamento (sentimentale) di concordia memoriale (vicino a quello dei dialoganti del conte Joseph De Maistre nelle sue famose Soirées de Saint-Pétersbourg) ${ }^{8}$, rimane inalterata, se non irrisolvibile, la discordia delle e sulle memorie. Nel contempo, tra i "reduci", è sempre in gioco la differenza che corre tra la "memoria passiva" che induce ad una mera nostalgia (inevitabilmente reazionaria) e una "memoria attiva" che del passato preserva le lezioni vitali per una tensione progressiva verso il futuro, accantonando quelle ormai superate, travolte e improponibili. Personalmente - preservando della mia tradizione metodi e valori e non un'astratta ortodossia dottrinale - mi sforzo di appartenere ai testimoni e agli esercenti della "memoria attiva", mentre una parte ancora piuttosto ampia dei miei compagni di generazione (e di loro tardivi seguaci) è così vittima di nostalgia da sperare di poter affrontare il futuro della rivoluzione del nuovo millennio ancora armata di categorie di giudizio, nonché di formule e propensioni utopiche, ormai inservibili o controproducenti. A questa condizione storico-esistenziale di "falsa dialettica" tra passato e

${ }^{8}$ DE MAISTRE, Joseph, Les soirees de Saint-Petersbourg, ou Entretiens sur le gouvernement temporel de la providence, Paris, Garnier, 1831. 
presente può ricondursi in gran parte anche il dramma dell'attuale Sinistra alla ricerca di una nuova, per adesso indecifrabile, identità culturale e politica. Certo è assai difficile adattarsi all'idea che il supercapitalismo stia di fatto liquidando la morfologia delle classi sviluppatasi nell'ormai superata età della "rivoluzione industriale"; convincersi del fatto che l'odierno esercito di poveri e di esclusi (sia nativi del sistema che migranti) non è il "proletariato"; che il "socialismo reale" è crollato soprattutto perché inadeguato alle sfide postmoderne della rivoluzione elettronico-informatica (come vorrei insistere a spiegare a Carmelo Albanese); che la dinamica dei nuovi conflitti sociali non è comparabile alla "lotta di classe" della manualistica marxiana; che i prodigiosi sviluppi della tecnologia (la proliferazione di robot ed algoritmi con i già prevedibili esiti epocali sui rapporti produzione-lavoro, fatica-tempo libero, società-libertà, ecc.) non siano più inquadrabili nella visione ottimistica con la quale Gramsci rifletteva sulle innovazioni del suo tempo in Americanismo e fordismo ${ }^{9}$, perché, adesso, non c'è tanto da esultare, come suggerisce Gozzini, per l'avanzata dell'intelligenza artificiale al fine di "rendere meno alienato il lavoro di fabbrica" (ma dov'è più la "fabbrica" nel senso classico e fordista?), quanto piuttosto da temere che l'uomo smetta di essere uomo; che la democrazia alla quale ancora pensiamo nelle forme ottocentesche sia destinata a non più funzionare nelle "società liquide" ben descritte da Bauman $^{10}$ (tra parentesi, se mi fosse consentito, vorrei rimproverare i miei commentatori per aver un po' eluso questo argomento, a parte l'attenzione rilevabile nel commento di Carmelo Albanese e nell'assai acuta ed esaustiva introduzione al dibattito di Fausto Pietrancosta). Almeno per adesso, il prodigioso "nuovo" che avanza nell'egemonia del capitalismo globalizzato segnato dall'ideologia neoliberista, per chi possieda lucida memoria, non è migliore del vecchio: anzi - rileva, amaro, Francesco Barbagallo - appare "di gran lunga peggiore della precedente forma del capitalismo fordista, che assicurava comunque alti salari, consumi di massa, integrazione sociale". Questo, mentre la corrente proposta "riformista", ovvero liberal-marxiana, di "un'alternativa non illuministica al neoliberismo che, stando dentro il mercato, costruisca la liberazione delle energie positive che stanno dentro gli esseri umani" (Giovanni Gozzini) sembra essere, piuttosto che una generica speranza, una tautologia: perché equivale a sperare che il capitalismo possa costituire l'alternativa di se stesso. Siamo, allora, caduti in un cul de sac? No. Stiamo vivendo una rivoluzione. C'è da ripeterlo ancora, quasi ossessivamente. Trovo sempre immane il compito di capirlo e di farlo capire persino alla cosiddetta Sinistra che, per sua tradizione, di rivoluzioni dovrebbe intendersene. Mi conforta che dell'enorme spessore culturale, tanto teoretico quanto di immediata valenza storica, della questione siano già pienamente avvertiti dei giovani come Paola Bernasconi (nel suo raffinato commento-saggio che ha valore di per se stesso, e reputo di integrazione al mio, a prescindere dalla sua contingente destinazione) e

\footnotetext{
${ }^{9}$ PLATONE, Felice (a cura di), Antonio Gramsci Americanismo e fordismo, Milano, Universale economica, 1949.

${ }^{10}$ Cfr. BAUMAN, Zygmunt, Modernità liquida, Roma-Bari, Laterza, 2002; ID., Vita liquida, Roma, Editori Laterza, 2008.
} 
come Carmelo Albanese ai cui sensati dubbi su alcuni punti della mia analisi vorrei rispondere in altra sede. Certo, per venire a capo della rivoluzione in corso disgregandone l'attuale egemonia totalitaria, alla Sinistra occorrerebbero a dir poco la genialità innovativa di un nuovo Marx e, a guida, la forza politica di un nuovo principe gramsciano, ovvero di un nuovo "intellettuale collettivo". Per adesso inimmaginabili. Potrà il mio pamphlet corsaro ben figurare almeno tra i prolegomeni di una "scienza della prassi" tutta da rinnovare? Se, dati i miei limiti, non sarà così, potrà comunque valere (sono solito immaginarlo) come uno di quei "messaggi" chiusi in una bottiglia che gli antichi naufraghi affidavano alle onde del mare. Il messaggio, quasi un testamento, di un passeggero del XX secolo al nuovo millennio già maturo. 


\section{L'AUTORE}

Giuseppe Carlo MARINO già ordinario di Storia Contemporanea nell'Università di Palermo, è autore - oltre che di noti studi sulla storia della Sicilia culminati nel best-seller internazionale Storia della mafia (Roma, Newton\&Compton, 1998, che vanta 12 ristampe) - di numerosi libri sui rapporti tra politica e società in Italia, tra i quali l'ormai classico $L a$ formazione dello spirito borghese in Italia (Firenze, La Nuova Italia, 1974), (con A. Consiglio Marchese) Liberi di non scegliere. Friedman e la frontiera neoliberale di Ronald Reagan (Palermo, Flaccovio, 1982), Autoritratto del Pci staliniano (Roma, Editori Riuniti, 1992), Guerra fredda e conflitto sociale in Italia (Caltanissetta-Roma, Sciascia, 1994) e altre opere di notevole successo editoriale quali La repubblica della forza (Milano, Franco Angeli, 1995), Eclissi del principe e crisi della storia (Milano, Franco Angeli, 2000), Biografia del Sessantotto (Milano, Bompiani, 2005), Saggio su De Gasperi, Togliatti e il trasformismo italiano (Firenze, Le Monnier, 2002), Le generazioni italiane dall'unità alla repubblica (Milano, Bompiani, 2008), Globalmafia (Milano, Bompiani, 2012) e, con Pietro Scaglione, L'altra Resistenza (Milano, Edizioni paoline, 2016).

URL: < http://www.studistorici.com/progett/autori/\#Marino > 